\title{
An adaptive radiation model for the origin of new gene functions.
}

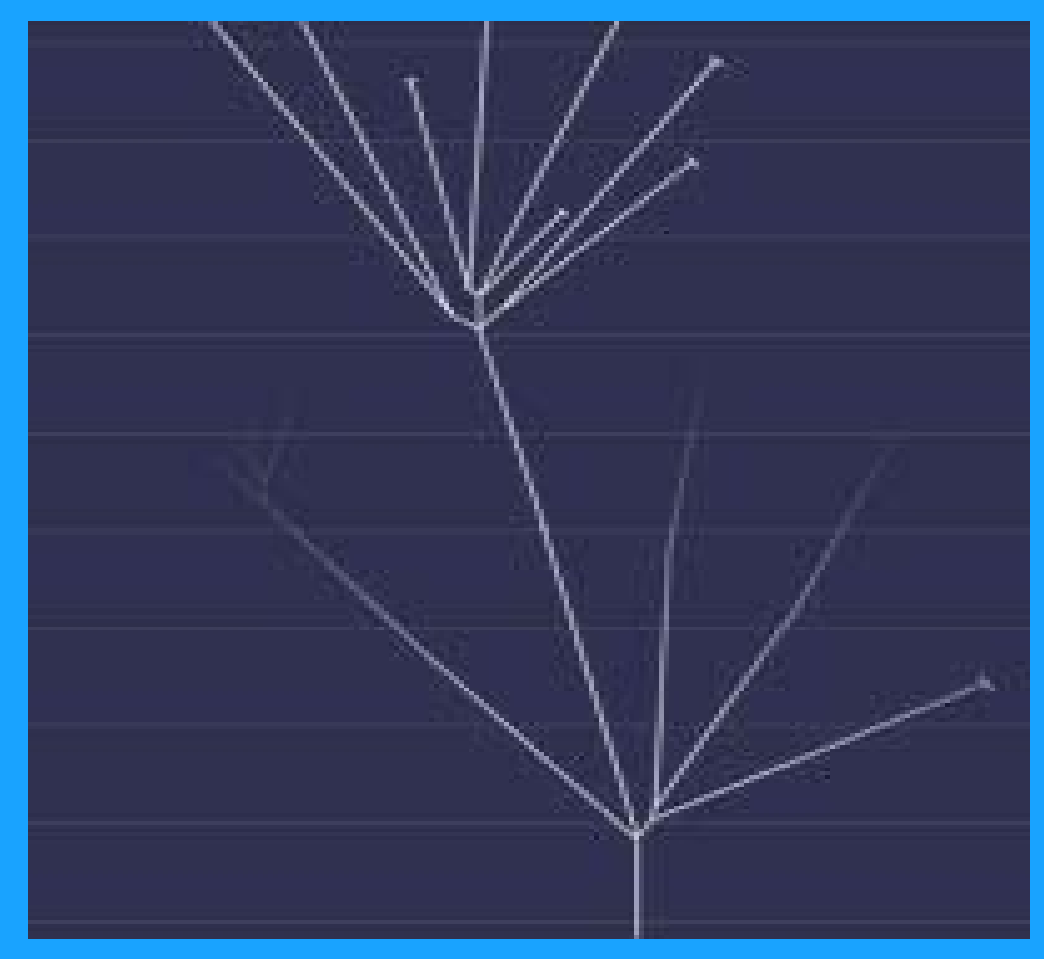




\section{Adaptation by Natural Selection}

Lineage diversification

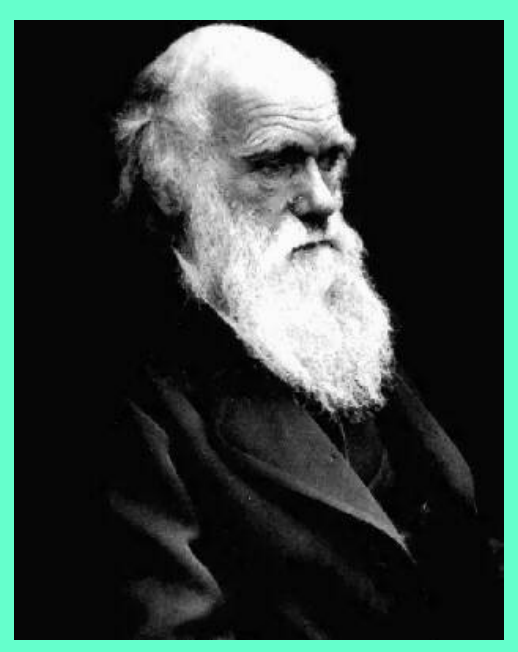



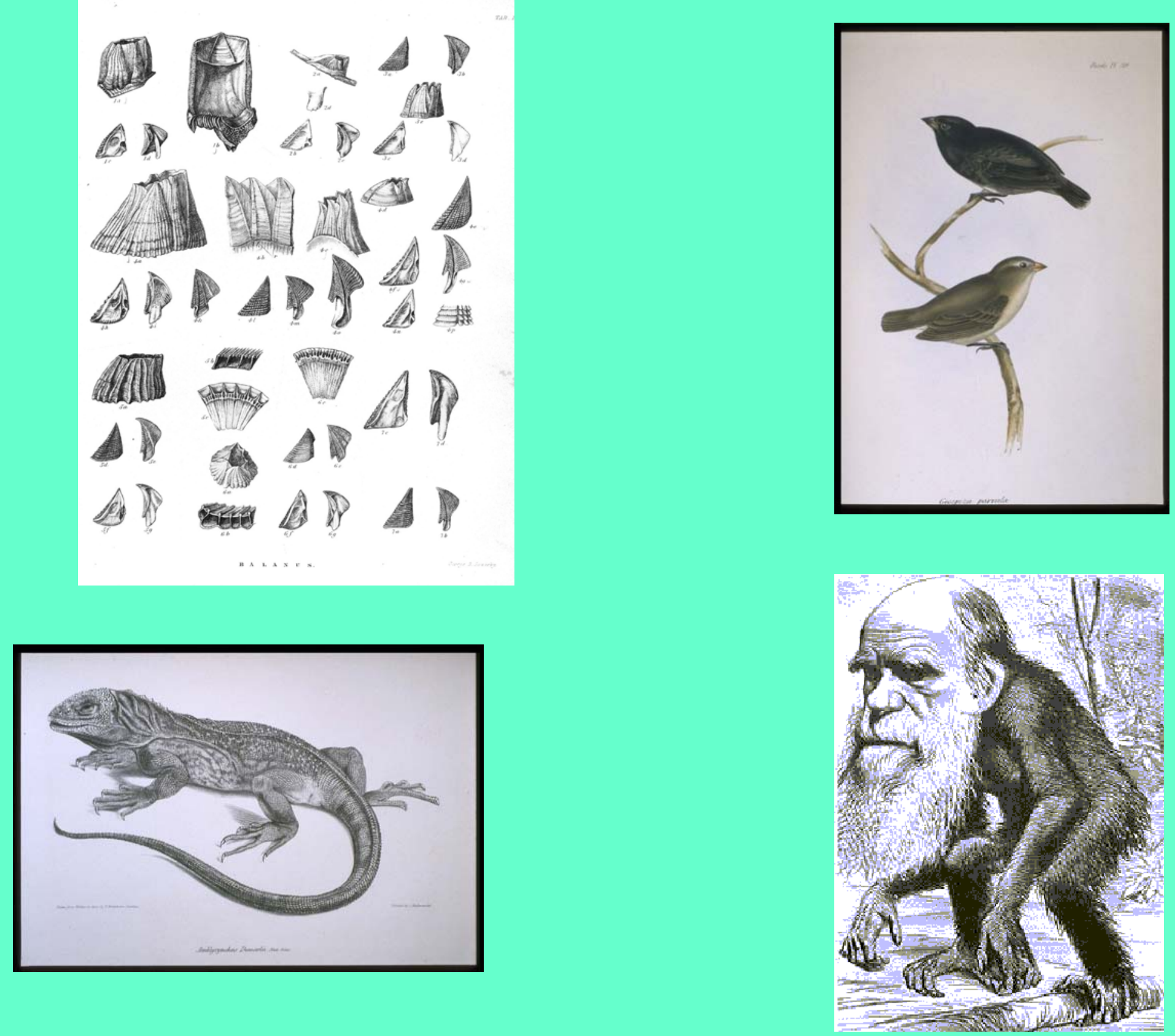


\section{Current Models}

Neofunctionalization Ohno 1970
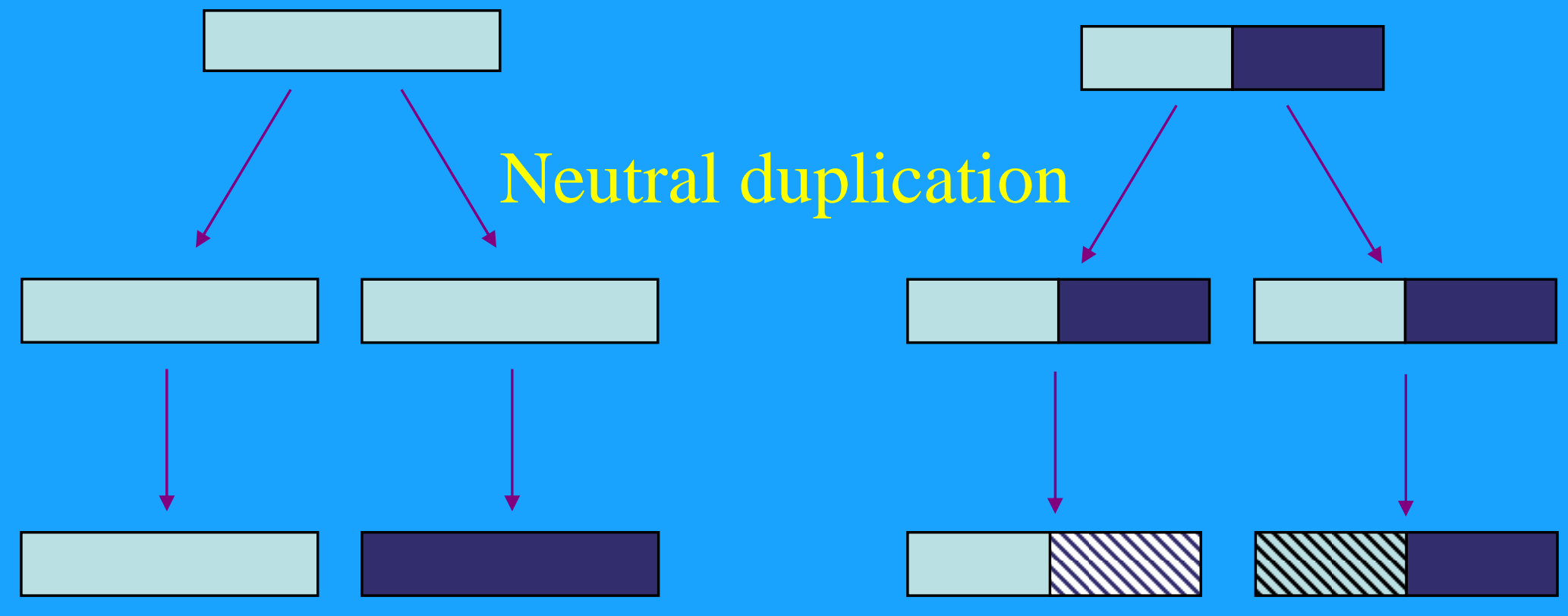

Neutral duplication

Subfunctionalization

Force et al. 1999 
New functions often arise from preadapted characters

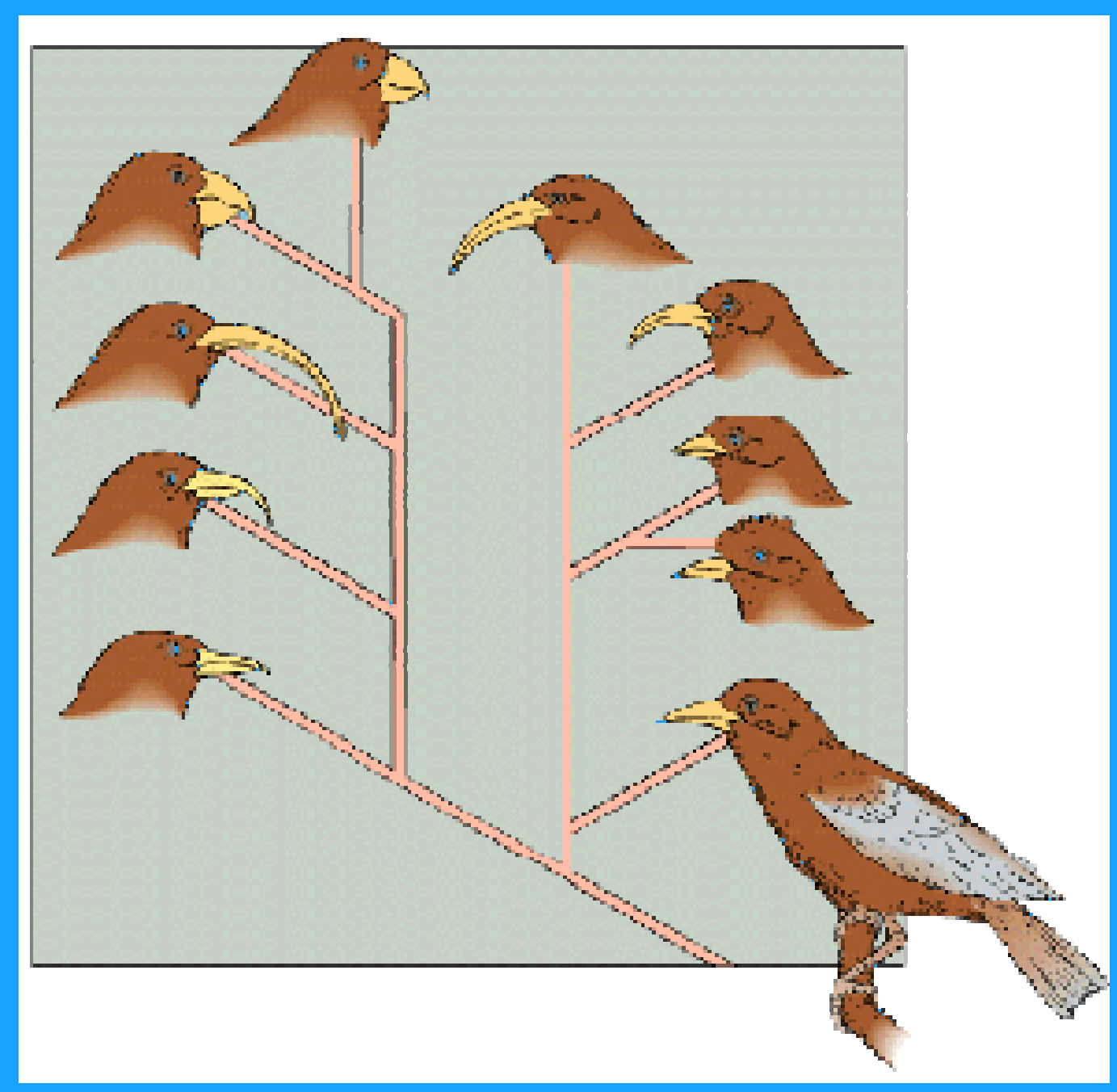


Ecological concepts:

Opportunity

Preadaptation

Adaptive radiation
Competition among close variants for a specific niche

Adaptive radiation
Competition among close variants for a specific niche

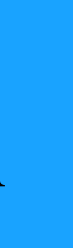 \\ tion
}




\section{Adaptive radiation model}
1)
\&
2)
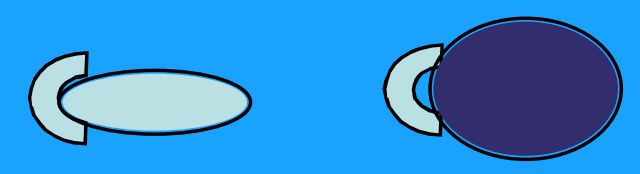
low binding affinity

Adaptive gene amplification

3)
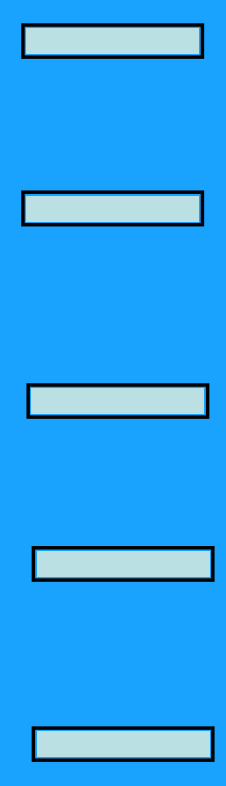
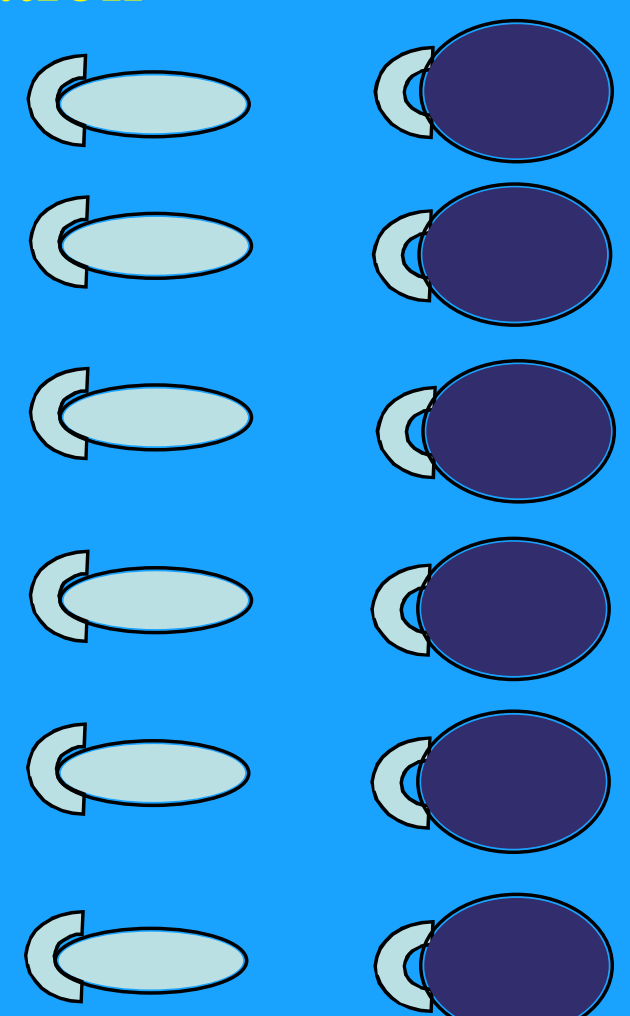
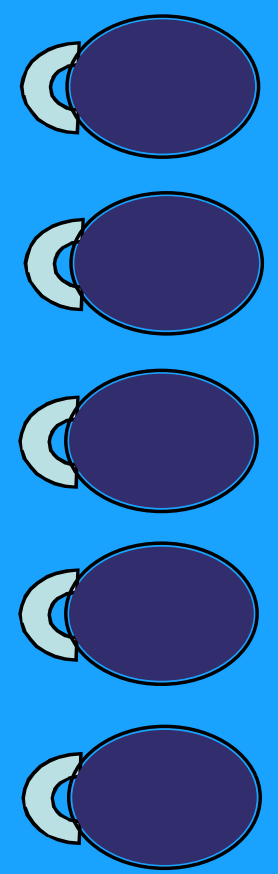


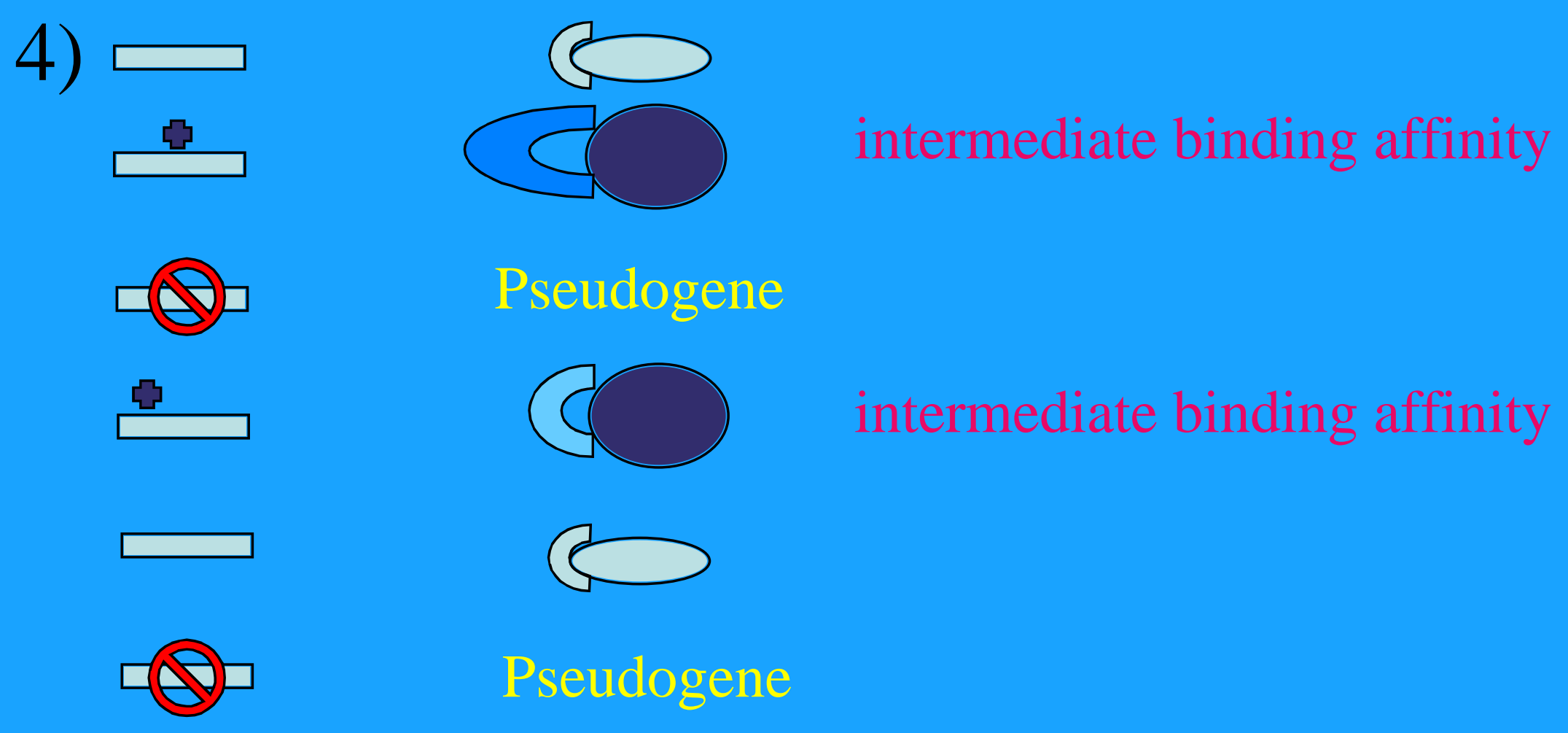

Adaptive mutations

Q Deleterious mutations 

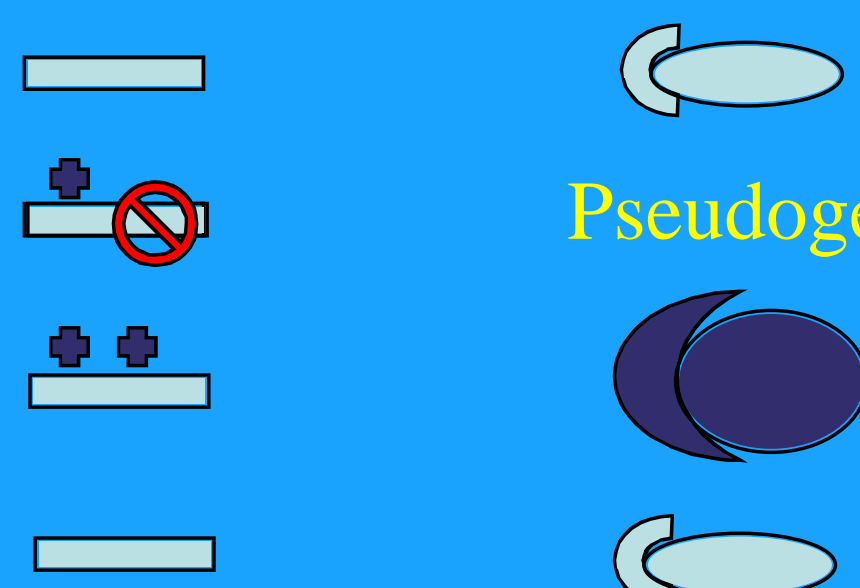

Pseudogene

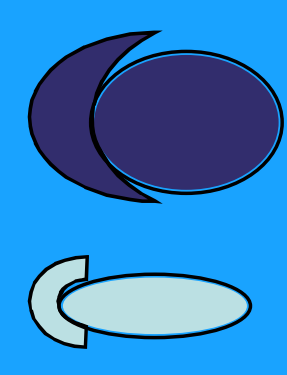

high binding affinity

Gene and pseudogene loss (neutral or adaptive)

6)

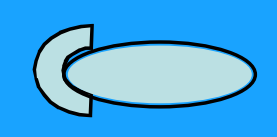

$\stackrel{5}{5}$

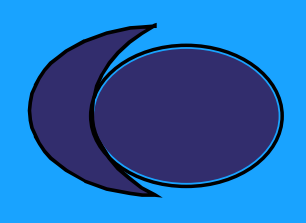




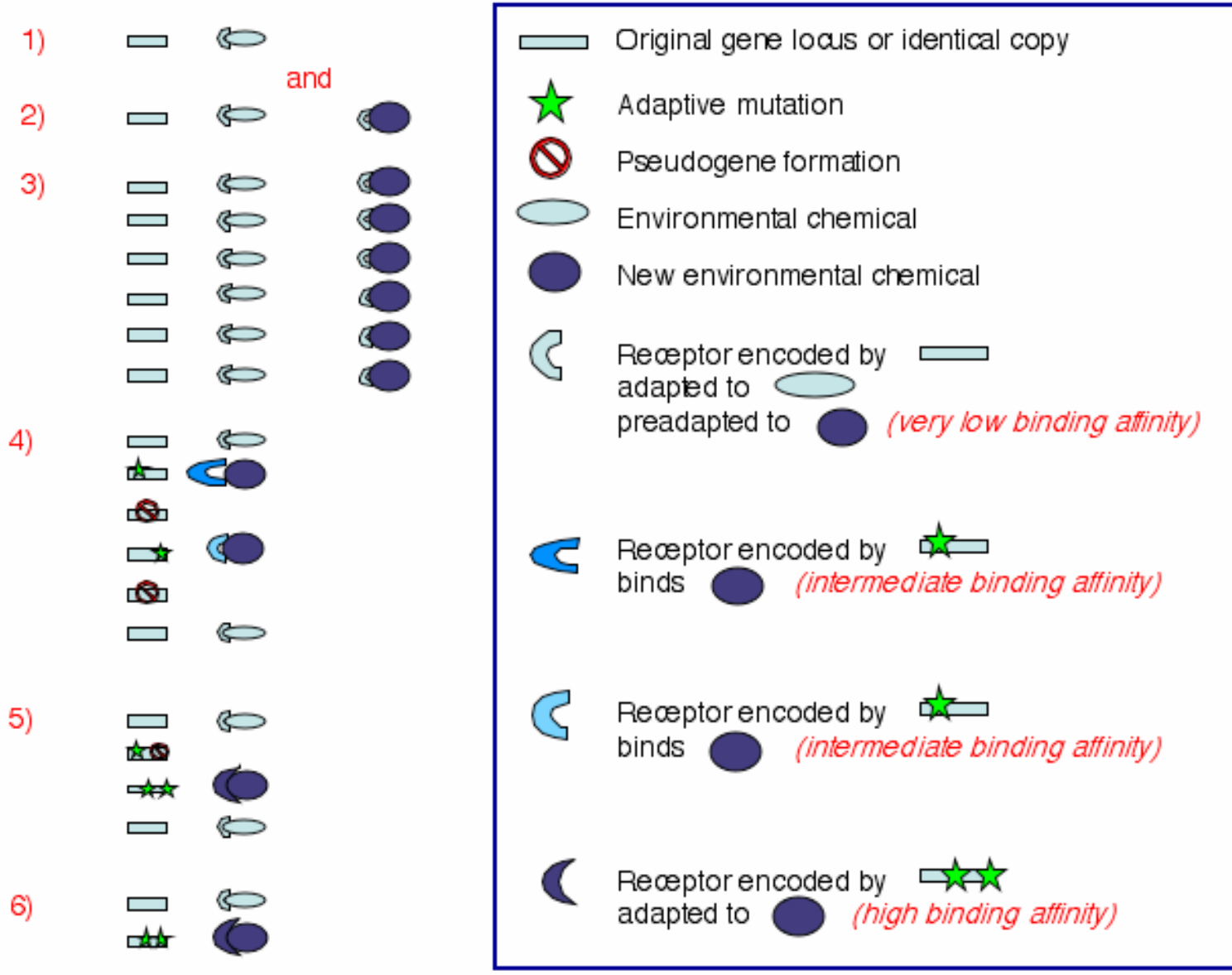

Figure 1 - Hypothetical course of events illustrating the adaptive radiation mode/for the evolution of new gene functions. 


\section{Advantages of the adaptive radiation model}

A selective advantage for gene amplification per se, followed by the evolution of new function

A multiplication of the probability of adaptive mutations due to the increased number of target sites

The potential exploration of multiple zones of the adaptive landscape by different gene copies, including fitness valleys

The potential recombination among gene copies to bring together adaptive mutations

The potential for sequential adquisitions of adaptive mutations, separated by rounds of amplification of the best adapted gene copies 
Selection experiments in bacteria produce transient gene amplifications (Hendrickson et al. 2002)

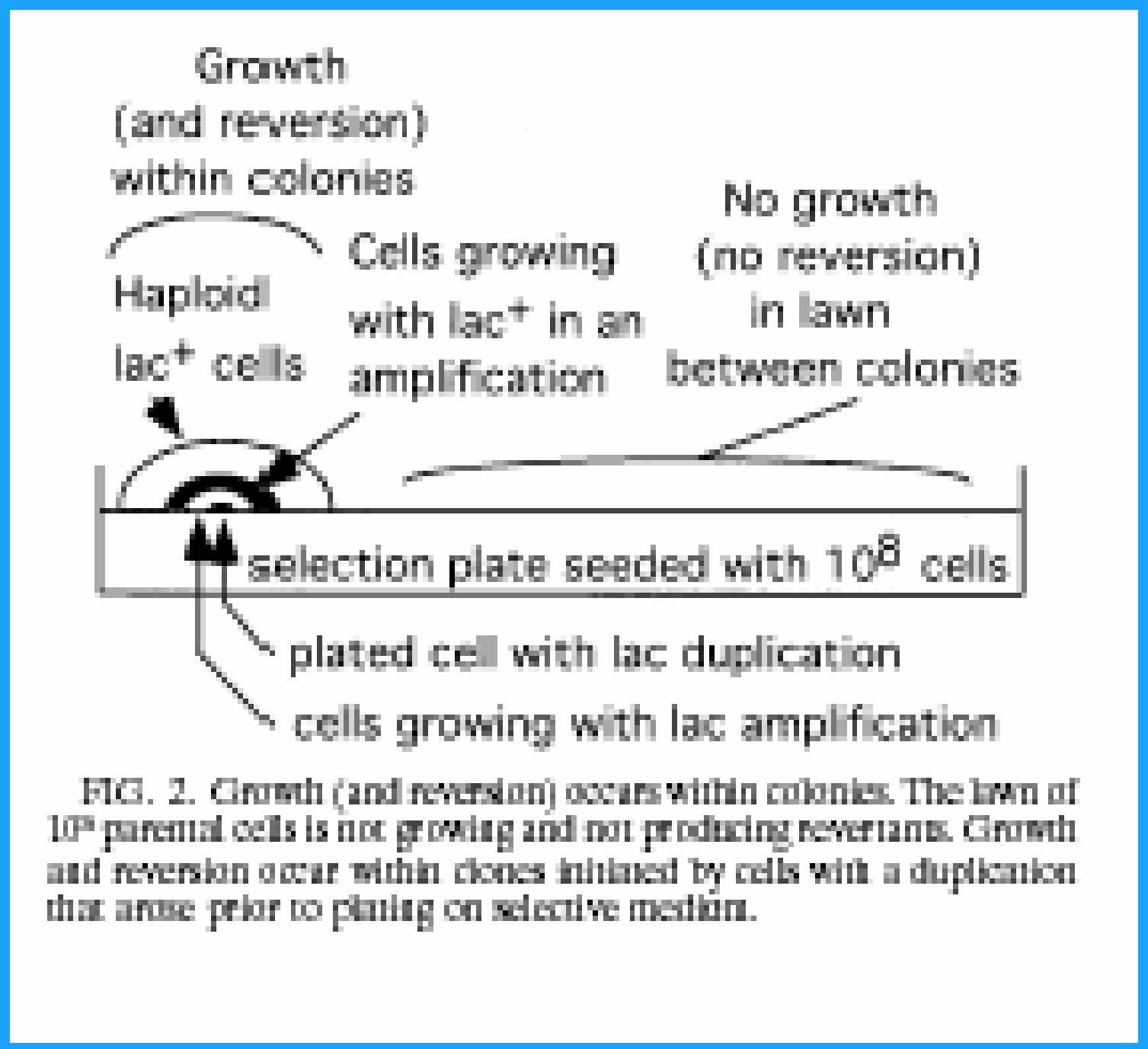




\section{Evidence from GENOMICS}

\section{1)Punctuated bursts of gene amplification and paralog fixation in response to specific selection pressures}

Gene duplications are most prevalent for functional classes known to be subject to frequent episodes of positive selection (Kondrashov et al 2002, Gevers et al. 2004)

The largest gene expansions are mostly confined to shallow phylogenetic depths (Lespinet et al. 2002)

- Power law distribution of gene family sizes indicating an overrepresentation of gene families that are much larger than the rest (Huynen and van Nimwegen 1998, Qian et al. 2001, Harrison and Gerstein 2002, Karev et al 2002, 2003) 


\section{Evidence from GENOMICS}

2) Early selection on paralogous gene copies

Genome-wide analyses show that retained paralogs do not undergo substantial periods of neutral evolution (Lynch and Connery 2000, Kondrashov et al. 2002)

Positive selection shortly after duplication detected in several analyses (Gilad et al. 2000 and 2003, Gilad and Lancet 2003, Clark et al. 2003, Zhang et al. 1998, Holloway and Begun 2004, Moore and Purugganan 2003)

Some pseudogenes have patterns of nucleotide substitution indicative of purifying selection before recent nonfunctionalization (Gilad et al. 2003, Newman and Trask 2003) 


\section{Evidence from GENOMICS}

3) Generation of numerous pseudogenes and eventual

\section{pseudogene loss}

Pseudogenes tend to be associated with environmental response families (Harrison and Gerstein 2002, Liu et al. 2004), which are subject to more variable selective pressures

Pseudogenes are more prevalent in gene families that have undergone organismic-specific expansions, indicative of recent selection (Harrison and Gerstein 2002)

High numbers of small protein motifs and pseudomotifs in intergenic regions (Harrison and Gerstein 2002), indicating decay of pseudogenes 


\section{Example: olfactory receptor genes (OR)}

1)Some OR subfamilies have duplicated so recently that their copy number, location and functionality of different copies are polymorphic within the human population (Trask et al. 1998, Mefford et al. 2001)

Some human functional OR genes have appeared in the very recent past. 
2) Some of the OR genes have patterns of nucleotide substitution (Gilad et al. 2000, 2003) and/or population polymorphism (Gilad et al. 2000, Clark et al. 2003, Gilad and Lancet 2003) indicative of positive natural selection, including some pseudogenes before recent non-functionalization (Gilad et al. 2003, Newman and Trask 2003)

Recent successful and unsuccessful attempts to produce novel functions in OR genes. 
3) The fraction of OR pseudogenes in humans is high when compared to other primates and rodents

(Gilad et al. 2003, Newman and Trask 2003)

\section{Under the adaptive radiation model,} high pseudogenization is expected during periods of proliferation of new functions in response to environmental change. 
Ongoing radiation of OR genes, rather than a simple degeneration of olfactory capabilities in the human lineage.

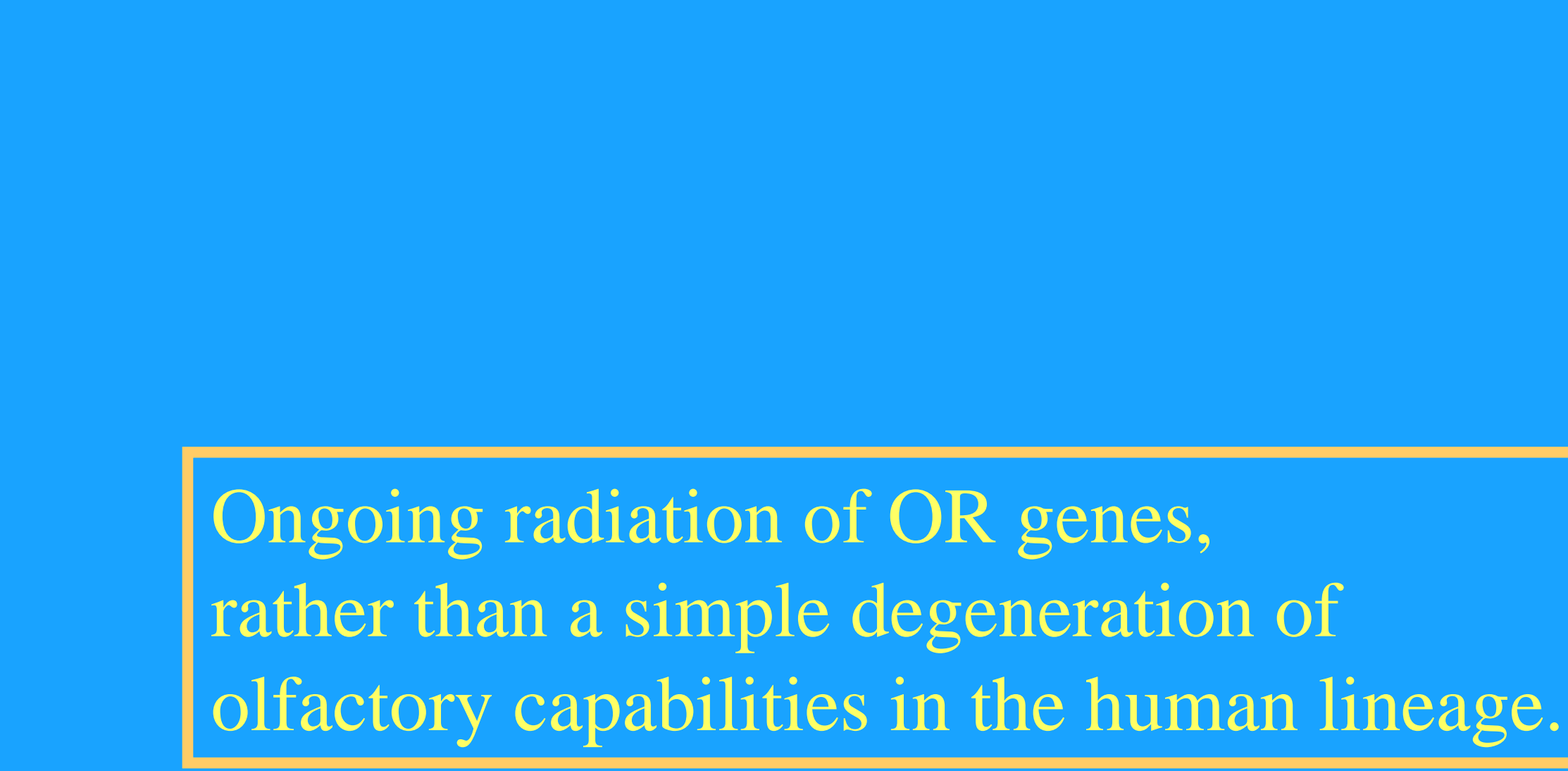




\section{Parallel to the macroevolution of organismal lineages}

\section{Punctuated Equilibrium}

observed patterns of change within lineages

(bursts of gene amplification accompanied by pseudogene formation)

hypothesis about evolutionary process

(rapid change associated with lineage/gene splitting, lineage selection, and extinctions of intermediate variants due to niche competition) 
The methodologies developed by paleontologists and evolutionary biologists to study rates and patterns of diversification of organismal lineages and the origins of evolutionary trends should be applicable to investigate the tempo and mode of gene family evolution, as well as the correlations of gene level events with organismal and environmental change. 


\section{ACKNOWLEDGEMENTS}

\section{JGI Evolutionary Genomics}

Monica Medina

Rick Baker

Jenna Morgan

Yvonne Valles

Martha Lucia Posada

Paramvir Dehal

Howard Ochman

Ulfar Bergthorsson

Gabriel Moreno-Hagelsieb

Eric Alm

Fyodor Kondrashov
University of Arizona University of New Mexico Wilfred Laurier University, Canada LBL, Arkin lab University of California Davis

Elbert Branscomb 
This work was performed under the auspices of the US Department of Energy's Office of Science, Biological and Environmental Research Program, and by the University of California, Lawrence Livermore National Laboratory under Contract No. W-7405-Eng-48, Lawrence Berkeley National Laboratory under Contract No. DE-AC02-05CH11231 and Los Alamos National Laboratory under Contract No. W-7405-ENG-36. 